\title{
FAST INTEGRAL EQUATION TECHNIQUES FOR PROPAGATION PROBLEMS
}

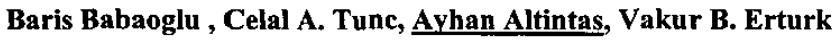 \\ Bilkent University, Electrical \& Electronics Engineering Department \\ Bilkent, 06800-Ankara, Turkey
}

\begin{abstract}
In this paper, the Method of Moments (MoM) solution is achieved for scattering problems by using the stationary Spectrally Accelerated Forward-Backward method (FBSA) and the non-stationary Spectrally Accelerated BiConjugate Gradient Stabilized (SA-BiCGSTAB) method, with a storage requirement and a computational cost of $O(N)$ per iteration where $N$ is the number of surface unknowns in the discretized integral equation. The SA-BiCGSTAB method is applied over rough terrain profiles as well as re-entrant surfaces which can not be handled by any conventional stationary iterative technique.
\end{abstract}

\section{INTRODUCTION}

Mobile radio planning requires accurate prediction of electromagnetic field strengths over large terrain profiles. For this purpose, a great number of solution techniques have been employed. The first class of these techniques is based on propagation prediction models. These are the automatic tools depending on empirical models used in conjunction with multiple- diffraction (MD) corrections [1] which proceed over geographical databases. The second class is the integral equation based methods. These techniques differ from the empirical type models by exposing the integral equations which then can be converted into a matrix equation by employing the Method of Moments (MoM) [2]. Conventional numerical solution methods to solve this matrix equation become not suitable for electrically large surfaces, because of the $O\left(N^{3}\right)$ computational cost due to the large number of surface unknowns $N$. The stationary iterative Forward-Backward Method (FBM) [3] and the non-stationary iterative BiConjugate Gradient Stabilized (BiCGSTAB) method [4] are techniques for solving linear equation systems resulting from electromagnetic rough surface scattering problems and providing accurate results within few iterations, causing a computational cost of $O\left(N^{2}\right)$. The Spectral Acceleration (SA) algorithm proposed in [5], utilized for very undulating rough geometries, reduced the computational cost and memory requirements to $O(N)$ which enables the use of the FBM over electrically large surfaces. The algorithm accelerates the matrix-vector multiplies taking place in the iterative technique.

An $e^{j \omega t}$ time harmonic convention is suppressed throughout the paper. The $\omega, k$ and $\lambda$ are the angular frequency, the wave number and the wavelength of the free space, respectively.

\section{IE AND MoM FORMULATION}

The terrain profile along the displacement ( $x$-axis) is characterized with the curve $C$ and defined by $z=f(x)$ as depicted in Fig. 1, yielding the roughness of the surface in one dimension. The electromagnetic fields characterized by $\mathbf{E}^{\mathrm{i}}(\boldsymbol{\rho})$ and $\mathbf{H}^{\mathrm{i}}(\boldsymbol{\rho})$, are incident upon the surface where $\boldsymbol{\rho}=\hat{x} x+\hat{z} z$. The terrain profile is modeled to be an imperfect conductor (with permeability $\mu$, and permittivity $\epsilon$ ) and analyzed using an impedance boundary condition (IBC) to be able to investigate more general situations.

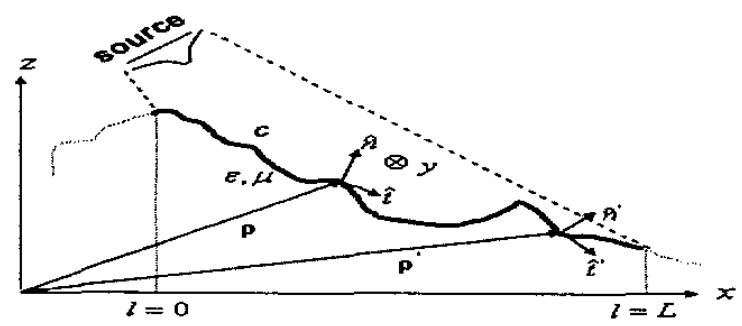

Fig.1. An arbitrary $1 D$ terrain profile. 
An IE is formed by applying boundary conditions on the surface of the scatterer. Using an IBC, the EFIE for a TM wave and MFIE for a TE wave can be written as

$$
\begin{aligned}
-E_{y}^{i}(\boldsymbol{\rho})= & -\eta_{s} J_{y}(\boldsymbol{\rho})-\frac{\omega \mu}{4} \int_{c} J_{y}\left(\boldsymbol{\rho}^{\prime}\right) H_{0}^{(2)}\left(k\left|\boldsymbol{\rho}-\boldsymbol{\rho}^{\prime}\right|\right) d \rho^{\prime} & \& & -H_{y}^{i}(\boldsymbol{\rho})=J_{t}(\boldsymbol{\rho})+j \frac{k}{4} \int_{c} J_{t}\left(\boldsymbol{\rho}^{\prime}\right) \hat{n}^{\prime} \cdot \hat{\mathbf{\rho}} H_{1}^{(2)}(k|\boldsymbol{\rho}-\boldsymbol{\rho}|) d \rho^{\prime} \\
& -j \frac{k \eta_{\mathrm{s}}}{4} \int_{c} J_{y}\left(\dot{\boldsymbol{\rho}}^{\prime}\right) \dot{\hat{n}}^{\prime} \cdot \hat{\boldsymbol{\rho}} H_{1}^{(2)}\left(k\left|\hat{\boldsymbol{\rho}}-\hat{\boldsymbol{\rho}}^{\prime}\right|\right) d \rho^{\prime} & & +\frac{\omega E \eta_{s}}{4} \int_{c} J_{t}(\boldsymbol{\rho}) H_{0}^{(2)}\left(k\left|\boldsymbol{\rho}-\boldsymbol{\rho}^{\prime}\right|\right) d \rho^{\prime},
\end{aligned}
$$

where $E_{y}^{i}$ and $H_{i}^{y}$ are the incident fields, $J_{y}$ and $J_{t}$ are the unknown current distributions to be solved which is tangential and transverse to the plane of the surface, respectively, $\eta_{s}$ is the surface impedance of the structure, $\rho$ and $\rho^{\prime}$ are the observation and source points, respectively, $\hat{n}^{\prime}$ is the unit normal vector at the source point, $\mathrm{C}$ is the contour of the surface and lastly, $H_{0}^{(2)}$ and $H_{1}^{(2)}$ are the Hankel functions of second kind with orders of zero and one, respectively. For the TE polarization case, MFIE is utilized.

The FBM is based on splitting the current at each point into two components: the forward contribution due to the incident field and the radiation of the current elements located in front of the receiving element and the backward contribution due to the current elements located beyond the receiving element. Non-stationary iterative BiCGSTAB method, converge to the exact solution by constructing orthogonal vector sequences. For both methods, the operational count is $O\left(N^{2}\right)$ per iteration, which prohibits the application of very large-scale scattering problems.

The spectral acceleration (SA) àlgorithm begins with selecting a neighborhood distance for a given receiving element. $N_{s}$ radiating elements within this neighborhood distance forms the strong group and their contribution to the receiving element is calculated through the matrix - vector products yielding a an operational cost of $O\left(N_{s}^{2}\right)$. The rest of the radiating elements form the weak group and the computation their contribution to the receiving element is accelerated based on using the spectral representation of the 2D Green's function yielding an $O\left(N-N_{s}\right)$ computational cost. The final field value for the receiving element is the sum of strong and weak contributions. Since $\left(N-N_{s}\right) \gg>N_{s}$, the total operational cost is still $O(N)$.

The convergence ability of the SA-FBM with an operation count and memory requirement of $O(N)$ makes the method to be applicable for the general use in the scattering problems. Unfortunately, if the target of interest has a re-entrant surface like breaking waves or a ship profile, the SA-FBM yields wrong results due to its stationary iterative nature. To overcome this deficiency, a generalized version of SA-FBM (GFBSA) was proposed in [6]. Still, electrically large re-èntrant surfaces prohibit the applicability of the SA-GFBM due to computational complexity. These complexities can be eliminated by using the SA-BiCGSTAB method whose convergence ability is independent of the ordering of surface of interest. Besides, since the BiCGSTAB method depend upon matrix-vector products, parallel implementation is possible.

\section{NUMERICAL RESULTS}

The example in Fig. 2 is a rough surface of width $20000 \lambda$ yielding 200000 unknowns at $300 \mathrm{MHz}$ to be solved in order to evaluate the scattered field for both polarizations. Imperfect conducting case with the surface impedance $\eta_{s}=20+j 15$ is considered. The surface is considered to be illuminated by a dipole antenna located at a $90 \lambda$ height above at the left most point of the terrain. The average radiated power is 90 Watts. As can be seen from Fig. 2, the agreement between the results is excellent.

In Fig. 3, propagation prediction models over a terrain profile, Cinarkoy, from Turkey are examined. The source is an isotropic radiator transmitting $50 \mathrm{~W}$ power at $890 \mathrm{MHz}$ operating frequency. It is located $20 \mathrm{~m}$ above the left-most point of the surface. Total field strength $1.8 \mathrm{~m}$ above the surface is obtained. SA-FBM results are compared with some of the widely used propagation prediction models. During these examinations, 600 thousand surface unknowns for the computation of rough surface scattering have been reached at $600 \mathrm{MHz}$.

The last example is an unordered surface profile. The target under test is a ship placed on a 400 meters sea surface. The electrical length properties with respect to $\lambda$ are illustrated in Fig. 4 . In order to show the validity of the SA-BiCGSTAB the results are compared with SA-GFBM. For the non-PEC case, the sea is modeled with an surface impedance $\eta_{s}=76.4+j 65.1$, while the ship still remains PEC. Plane wave incidence is considered with an incident angle $\theta=\pi / 36$. Current distributions both on the surface of the sea and the ship due to $5 \mathrm{~m} / \mathrm{s}$ wind 
MMU $10^{\text {th }}$ Int. Conf, on Mathematical Methods in Electromagnetic Theory

speed is plotted in Fig. 5.The frequency is $300 \mathrm{MHz}$ yielding the number of unknowns about $N=4500$. The results agree very well.

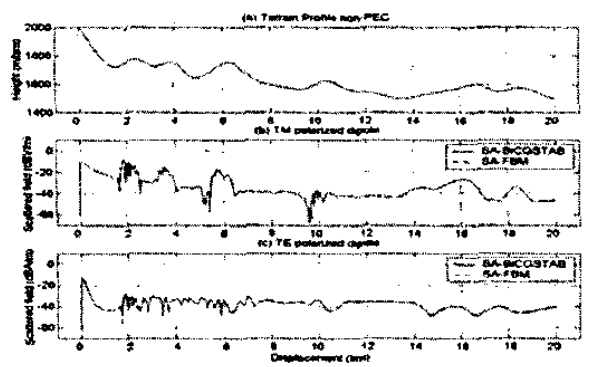

Fig. 2. Scattered fields from a rough surface at $300 \mathrm{MHz}$.

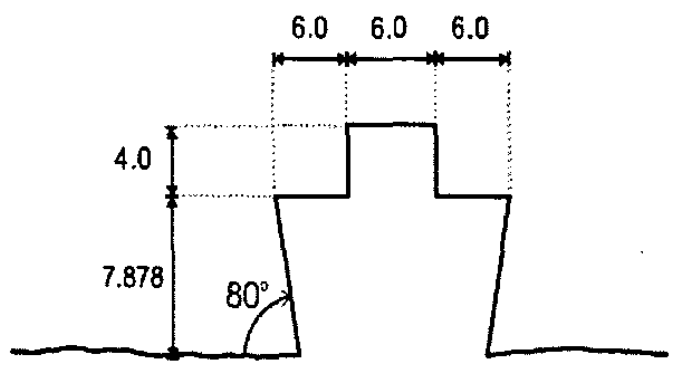

Fig. 4. Electrical length properties of the ship.

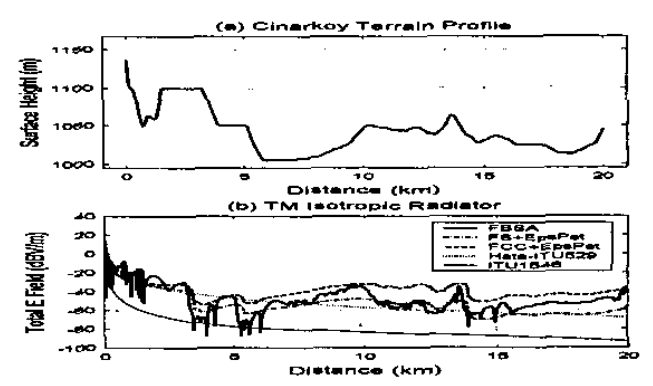

Fig. 3. Propagation models v.s SA-FBM.

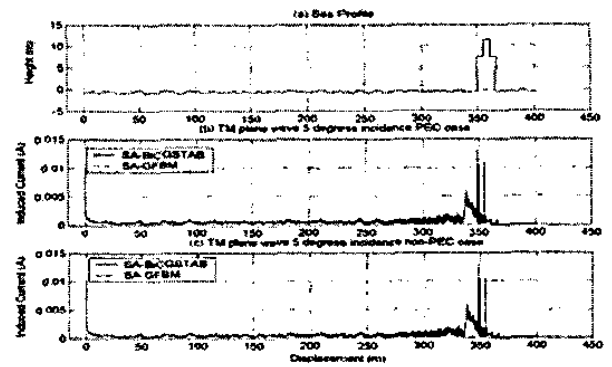

Fig. 5. Current distributions SA-BiCGSTAB vs. SA-GFBM.

\section{CONCLUSION}

In this work, fast integral equation based numerical methods are employed for the analysis of electrically large scattering problems and shown reliable predictions can be achieved. The accuracy of most widely used empirical models with MD corrections is investigated using SA-FBM as a reference solution. Another innovation is the examination of multi-valued surface profiles with a non-stationary technique without any computational complexity or high memory requirements. The variants of stationary techniques developed to treat these kinds of profiles such as SA-GFBM cannot compete with SA-BiCGSTAB method when the reentrant surface is electrically large.

\section{REFERENCES}

[1] M. Hata, "Empirical formula for propagation loss on mobile radio services," IEEE Trans. Veh.Tech., vol. 29, Aug. 1980.

[2] R.F. Harrington, Field Computation by Moment Method, New York: IEEE Press, 1993.

[3] D. Holliday, L. L. DeRaad Jr., and G. J. St.-Cyr, "Forward-Backward: A new method for computing low-grazing scattering," IEEE Trans. Antennas Propagat., vol. 44, pp. 722-729, May 1996.

[4] R. Barret, M. Berry, T. F. Chan, J. Demmel, J. Donato, J. Dongarra, V. Eijkhout, R. Poza, C. Romine, and H. Ban der Vort, "Templates for the Solution of Linear Systems," Philadelphia, PA: SIAM, 1994.

[5] J. A. Lopez, M. R. Pino, F. Obelleiro, J. L. Rodriguez, "Application of the spectral acceleration forward-backward method to coverage analysis over terrain profiles," J. of Electromagn. Waves and Appl., vol. 15, no. 8, pp. 1049-1074, 2001.

[6] M. R. Pino, R. J. Burkholder, and F. Obelleiro, "Spectral Acceleration of the Generalized Forward-Backward Method," IEEE Trans. Antennas Propagat., vol. 50, no. 6, June 2002. 